\title{
COMPARATIVE EVALUATION OF THE ALLERGAN HUMPHREY 570 AND CANON RK-1 AUTOREFRACTORS: I. OBJECTIVE AUTOREFRACTION IN NORMAL SUBJECTS
}

\author{
PALANISWAMY SUNDER RAJ, JOSE R. VILLADA, ANNE E. LEWIS, PATRICK W. JOYCE, \\ ALLAN WATSON \\ Southport
}

\begin{abstract}
SUMMARY
Fifty normal subjects were studied comparing objective autorefraction using the Allergan Humphrey 570 (AH 570) and Canon RK-1 autorefractors in terms of ease of operation, the time taken and their accuracy compared with clinical refraction. Both the autorefractors were equally easy to operate but the AH 570 was quicker. Objective autorefraction with the AH 570 was more accurate than using the Canon RK-1 especially with respect to spherical equivalence, sphere power and cyclinder axis: approximately $80 \%$ of the values were within 0.51 dioptres or $11^{\circ}$ of clinical refraction compared to approximately $60 \%$ for the Canon RK-1. The possible reasons for the superior performance of the AH 570 are discussed.
\end{abstract}

Since the introduction of the first fully automated refractor (the ophthalmetron) in $1970,{ }^{1}$ a variety of new autorefractors have become commercially available, offering improved speed, efficiency, accuracy or ease of operation. $^{2}$

The Canon RK-1 which has an autofogging mechanism to relax accommodation combines both automated refraction (AR) and automated keratometry in one instrument. It also has an intraocular lens (IOL) setting to improve the accuracy of AR in pseudophakes. Studies in normal subjects ${ }^{2}$ and pseudophakes ${ }^{4,5}$ have shown that acceptably accurate objective refraction can be obtained with this instrument.

The Allergan Humphrey 570 (AH 570) is one of a small number of modern autorefractors which have the facility for both objective and subjective refraction. Other special features include an optional autofogging mechanism to

From: Department of Ophthalmology, District General Hospital, Southport PR8 6NJ.

Correspondence to: Mr P. Sunder Raj FCOphth FRCS, Department of Ophthalmology, District General Hospital, Southport PR8 6NJ. relax accommodation, and an automatic fine alignment system which tracks small eye movements giving constant centration of the pupil. Although studies with other Allergan-Humphrey models have demonstrated acceptable accuracy in normal subjects, ${ }^{2,6}$ to our knowledge no published information is available for the AH 570.

This study was performed in normal subjects firstly to compare the accuracy of objective AR using the AH 570 and Canon RK-1, and secondly to examine whether the automatic alignment system present in the AH 570 provides any advantage over the Canon RK-1 in terms of ease and efficiency of operation.

\section{PATIENTS AND METHODS}

\section{Instruments}

The principle of objective AR, the facilities possessed and the actual measurement of refraction with the Canon RK-1 have been described in detail in a previous paper. ${ }^{4}$

The AH 570 uses a near infra-red light of $880 \mathrm{~mm}$ and investigates all power meridians simultaneously using the knife-edge principle with an optical null detector. Variable power sphero-cylindrical optics are driven until the best correction is found.

The AH 570 requires a minimum pupillary area of at least $2 \mathrm{~mm}$ for measurement purposes although $3.2 \mathrm{~nm}$ is recommended. ${ }^{2}$ It can measure from +20 dioptres (D) to $-12 \mathrm{D}$ sphere, up to $6 \mathrm{D}$ cylinder, and axis between $1^{\circ}$ and $180^{\circ}$ in increments of $0.125 \mathrm{D}$ and $1^{\circ}$ respectively. The vertex distances can be set at $0.0 \mathrm{~mm}$ or between $10.5 \mathrm{~mm}$ and $16.5 \mathrm{~mm}$ in steps of $1.5 \mathrm{~mm}$. It has an optional autofogging mechanism which can be used to relax accommodation. The visual acuity can be measured with several interchangeable internally illuminated Snellen charts. Subjective verification of the objective AR value is possible using the lenses within the instrument.

After initial alignment of the autorefractor with the 
patient's eye by the operator the AH 570 makes an automatic vertex adjustment and then continues to make automatic fine alignment adjustments to compensate for eye movement. If the optical axis cannot be tracked due to patient movement this is indicated by the machine and the patient can be repositioned. Each objective AR cycle, during which a series of measurements are taken, lasts between five seconds (without autofogging) and 15 seconds (with autofogging). The 'standardised' AR value is displayed and can be printed out.

\section{Patients}

This was a prospective study carried out on healthy adults without any ocular pathology. All the clinical refractions were carried out by the same examiner (PSR) without the use of either mydriatics or cycloplegics. Streak retinoscopy was first carried out followed by subjective verification to achieve the best possible visual acuity.

The first 25 subjects underwent objective AR with the AH 570 by the second author (JRV) and with the Canon RK-1 by the third author (AEL, a senior nurse); the roles of these examiners were reversed for the last 25 subjects. Since the Canon RK-1 has an inbuilt autofogging mechanism, the optional autofogging system in the AH 570 was switched on in all subjects. The three examiners were not aware of the results obtained by one another. The standard refractive value of the left eye in the autorefractor $\mathrm{AH}$ 570/Canon RK-1) print-out was compared with the final prescription based on clinical refraction (CR) of the left eye for degree of agreement.

The ease of operation with the autorefractors was subjectively assessed by the operators (JRV, AEL) using the following scale: 1 : very difficult, 2 : fairly difficult, 3 : fairly easy and 4 : very easy.

The time taken to perform objective AR was estimated with an electronic stopwatch from the time the subject placed his/her chin on the chin rest to the completion of the AR print out. Both eyes were studied to enable evaluation of the special facility present in the $\mathrm{AH} 570$ by which it automatically moves to the left eye after refracting the right eye. Only one eye per subject, the left eye, was used to determine accuracy and ease of operation for statistical purposes. ${ }^{7}$

\section{Statistical Analysis}

The statistical significance of the differences between the AH 570 and the Canon RK-1 autorefractors was evaluated using:

Table I. Mean differences between autorefraction and clinical refraction

\begin{tabular}{lcc}
\hline Refractive Component & $\begin{array}{c}\text { Mean difference* (Standard deviation) } \\
\text { Allergan Humphrey 570 }\end{array}$ & Canon RK-1 \\
\hline Spherical equivalence (D) & $-0.14(0.81)$ & $-0.26(0.72)$ \\
Sphere power (D) & $-0.19(0.77)$ & $-0.28(0.73)$ \\
Cylinder power (D) & $-0.01(0.50)$ & $0.02(0.56)$ \\
Cylinder axis (degrees) & $8.7(9.2)$ & $9.9(9.2)$ \\
\hline
\end{tabular}

*the minus (-) sign indicates more myopia or less hypermetropia on autorefraction (AR) than by clinical refraction (CR) (i) Student's ' $t$ ' test for ease of operation, time taken to perform objective AR and the mean difference in the various refractive components between $A R$ and $C R$

(ii) chi-squared $\left(\mathrm{X}^{2}\right)$ test on the number of subjects (eyes) in the two groups within $0.51 \mathrm{D}$ of spherical equivalence, sphere, power or cylinder power and within $11^{\circ}$ in cylinder axis as determined by CR.

\section{RESULTS}

Fifty one adults with healthy eyes and not wearing contact lenses took part in this study. A valid AR was not possible with the Canon RK-1 in one subject for unknown reasons and this subject (eye) was excluded from further analysis.

The ages of the 50 subjects included in the study ranged from 19 to 64 years with a mean of 41 (SD 11) years. There were 21 males and 29 females. The refractive errors of the subjects on clinical refraction ranged from $-13 \mathrm{D}$ to $+5.75 \mathrm{D}$ in spherical equivalence with a mean of -1.47 (SD 3.7) D.

The ease of operation with the AH 570 was grade 3 or 4 with a mean of 3.9 (SD 0.3): ease of operation with the Canon RK-1 ranged from 1 to 4 with a mean of 3.8 (SD 0.5 ). This difference was not statistically significant $(\mathrm{p}>0.1)$.

The time taken to perform objective $\mathrm{AR}$ for each subject (both eyes) with the AH 570 varied from 42 seconds to 2 minutes 5 seconds ( 125 seconds) with a mean of 53 (SD 15) seconds: with the Canon RK-1 this was between 45 seconds and 4 minutes 20 seconds ( 260 seconds) with a mean of 71 (SD 52) seconds. This difference was statistically significant $(\mathrm{p}<0.01)$.

With the AH 570 twenty eyes were ascribed a cylinder value on objective $A R$ which was not confirmed with $C R$. With the Canon RK-1 twenty eyes were ascribed a cylinder value on objective $A R$ which was not present with CR: the reverse was true in two eyes. Only eyes which had a cylinder value on both AR and CR (30 eyes with the AH 570 and 28 with Canon RK-1) were used in evaluating the accuracy of the autorefractors in determining the cylindrical axis.

Table I shows the mean differences between AR and $\mathrm{CR}$ for the various refractive components. The spherical

Table II. Percentage agreement between autorefraction and clinical refraction for the various refractive components

\begin{tabular}{lccc}
\hline Refractive Component & $\begin{array}{c}\text { Agreement between Autorefraction and } \\
\text { Clinical refraction (in cumulative \%) }\end{array}$ \\
\hline & $\begin{array}{c} \pm 0.25 \mathrm{D} \\
\pm 5^{\circ}\end{array}$ & $\begin{array}{c} \pm 0.5 \mathrm{D} \\
\pm 10^{\circ}\end{array}$ & $\begin{array}{c} \pm 1.0 \mathrm{D} \\
\pm 20^{\circ}\end{array}$ \\
Spherical equivalence $(\mathrm{n}=50)$ & & & \\
AH 570 & 56 & 78 & 98 \\
Canon RK-1 & 40 & 62 & 88 \\
Sphere power $(\mathrm{n}=50)$ & & & \\
AH 570 & 50 & 76 & 98 \\
Canon RK-1 & 44 & 64 & 94 \\
Cylinder power $(\mathrm{n}=50)$ & & & \\
AH 570 & 60 & 82 & 100 \\
Canon RK-1 & 52 & 78 & 98 \\
Cylinder axis & & & 97 \\
AH 570 $(\mathrm{n}=30)$ & 57 & 80 & 86 \\
Canon RK-1 $(\mathrm{n}=28)$ & 43 & 64 & 97 \\
\hline
\end{tabular}


equivalence and sphere power were more myopic or less hypermetropic with AR compared with $\mathrm{CR}$. The mean differences between the two autorefractors for the various refractive components studied were not statistically significant (student's ' $t$ ' test: $p>0.1$ for spherical equivalence and sphere power, $p>0.3$ for cylinder axis and $p>0.5$ for cylinder power).

The percentage agreement between $A R$ and $C R$ for the various refractive components is depicted in Table II. The differences between the AH 570 and Canon RK-1 were not statistically significant $\left(x^{2}\right.$ test: $p>0.05$ for spherical equivalence and cylinder axis, $\mathrm{p}>0.1$ for sphere power and $p>0.5$ for cylinder power).

\section{DISCUSSION}

This study demonstrates that both the Canon RK-1 and Allergan Humphrey 570 autorefractors offer rapid and reasonably accurate ${ }^{9}$ objective refraction of normal eyes. The AH 570 was significantly quicker than the Canon RK-1.

Both the autorefractors produced some degree of instrument-induced erroneous myopia, possibly due to inadequacy of their autofogging machanisms. ${ }^{8}$ The degree of error was small, and was less with the AH-570 than the RK-1 although the difference was neither statistically nor clinically significant:

In approximately $40 \%$ of the eyes studied both the autorefractors detected a cylinder value which was not found to be clinically significant by conventional refraction. This confirms the reported tendency of autorefractors to detect clinically insignificant cylinder values in normal subjects. $^{4,5,9}$

The mean differences in the various components of refraction and the percentage agreement between autorefraction and clinical refraction did not reveal any statistically significant difference in accuracy between AH 570 and Canon RK-1. The spherical equivalent, sphere power and cylinder axis were within $0.51 \mathrm{D}$ or $11^{\circ}$ of clinical values for $80 \%$ of the results from the $\mathrm{AH} 570$ and $60 \%$ of those from the Canon RK-1. This difference was not statistically significant probably due to the small number of eyes studied (50). However, in our opinion this difference between the two autorefractors was clinically significant:

The AH 570 was more accurate than the Canon RK-1 probably because

(i) its automatic fine adjustment system gives constant centration of the pupil; this has to be attempted manually in the Canon RK-1 possibly resulting in some slightly off-axis readings. (ii) the AH 570 investigates all power meridians simultaneously while the Canon RK-1 calculates the cylinder from measurements in three fixed meridians, leading to lower accuracy in axis determination.

(iii) the vertex distance is automatically adjusted for each subject by the AH 570 while the Canon RK-1 uses a fixed vertex distance.

The objective refraction values obtained with both the autorefractors studied indicate that as previously recommended subjective verification is essential before final prescription of spectacles. ${ }^{2,4,5}$ An autorefractor which allows subjective refinement of objective AR values through built-in lenses and test charts (as in the AH 570) may provide an advantage over autorefractors which can only perform objective AR.

This study confirmed the high degree of accuracy offered by autorefractors. It was found that the more highly automated of the two instruments tested (the $\mathrm{AH}$ 570) was both quicker and more accurate in normal subjects. This is of particular significance where the machine is to be operated by a nurse or technician in advance of the ophthalmologist or optometrist confirming the refraction. A further study is in progress to assess the value of the facility to subjectively verify the objective AR with the AH 570.

Key words: Automated refraction, Autorefraction, Clinical refraction.

\section{REFERENCES}

1. Safir A, Knoll HA, Mohrmann R: Automatic objective refraction Trans Am Acad Ophthalmol Otolaryngol 1970, 74: 1266-75

2. Wesemann W and Rassow B: Automatic infra-red refractors-a comparative study Am J Optom Physiol Opt 1987, 64: 627-38.

3. Ehrlich DL: The Canon RK-1. Refractor mode evaluation Ophthalmic Physiol Opt 1990, 10: 97.

4. Sunder Raj P, Akingbehin T, Levy AM: Objective autorefraction in posterior chamber psuedophakia $\mathrm{Br} J$ Ophthalmol 1990, 74: 731-3.

5. Sunder Raj P, Villada JR, Myint K, Lewis AE, Akingbehin T: Clinical evaluation of automated refraction in anterior chamber pseudophakia Br J Ophthalmol 1991, 75: 42-4.

6. Wong EK, Patella MV, Pratt MV, Myers SW, Gaster RN, Leopold IH: Clinical evaluation of the Humphrey autorefractor Arch ophthalmol 1984, 102: 870-5.

7. Newcombe RG and Duff GR: Eyes or patients? Traps for the unwary in the statistical analysis of ophthalmological studies Br J Ophthalmol 1987, 71: 645-6.

8. Nayak BK, Ghose S, Singh JP: A comparison of cyloplegic and manifest refractions on the NR-1000F (an objective autorefractometer) $\mathrm{Br} J$ Ophthalmol 1987, 71: 73-5.

9. Ghose S, Nayak BK, Singh JP: Critical evaluation of the NR-1000F autorefractometer Br J Ophthalmol 1986, 70: 221-6. 